Prons

trobertivier Journal of Nonlinear Mathematical Physics

\title{
A three-component Camassa-Holm system with cubic nonlinearity and peakons
}

Baoqiang Xia, Ruguang Zhou, Zhijun Qiao

To cite this article: Baoqiang Xia, Ruguang Zhou, Zhijun Qiao (2015) A three-component Camassa-Holm system with cubic nonlinearity and peakons, Journal of Nonlinear Mathematical Physics 22:1, 155-169, DOI:

https://doi.org/10.1080/14029251.2015.996446

To link to this article: https://doi.org/10.1080/14029251.2015.996446

Published online: 04 January 2021 


\title{
A three-component Camassa-Holm system with cubic nonlinearity and peakons
}

\author{
Baoqiang Xia \\ School of Mathematics and Statistics, Jiangsu Normal University \\ Xuzhou, Jiangsu 221116, P.R. China \\ xiabaoqiang@126.com \\ Ruguang Zhou \\ School of Mathematics and Statistics, Jiangsu Normal University \\ Xuzhou, Jiangsu 221116, P.R. China \\ zhouruguang@jsnu.edu.cn \\ Zhijun Qiao \\ Department of Mathematics, University of Texas-Pan American \\ Edinburg, Texas 78541, USA \\ qiao@utpa.edu
}

Received 29 July 2014

Accepted 23 October 2014

\begin{abstract}
In this paper, we propose a three-component Camassa-Holm $(3 \mathrm{CH})$ system with cubic nonlinearity and peaked solitons (peakons). The $3 \mathrm{CH}$ model is proven to be integrable in the sense of Lax pair, Hamiltonian structure, and conservation laws. We show that this system admits peakons and multi-peakon solutions. Additionally, reductions of the $3 \mathrm{CH}$ system are investigated so that a new integrable perturbed $\mathrm{CH}$ equation with cubic nonlinearity is generated to possess peakon solutions.
\end{abstract}

Keywords: Three-component Camassa-Holm equation; Peakon; Lax pair; Conservation laws.

2000 Mathematics Subject Classification: 37K10, 35Q51

\section{Introduction}

In the past two decades, the Camassa-Holm $(\mathrm{CH})$ equation [4]

$$
m_{t}-2 m u_{x}-m_{x} u=0, \quad m=u-u_{x x}+k,
$$

with $k$ being an arbitrary constant, derived by Camassa and Holm [4] as a shallow water wave model, has attracted much attention in the theory of soliton and integrable system. The $\mathrm{CH}$ equation was first included in the work of Fuchssteiner and Fokas on hereditary symmetries as a very special case [18]. Since the work of Camassa and Holm [4], more diverse studies on this equation have remarkably been developed $[2,5,7,10,13,15,16,20,23,28,32,33]$. The most interesting feature of the $\mathrm{CH}$ equation (1.1) is that it admits peaked soliton (peakon) solutions in the case $k=0$. A peakon is a kind of weak solution in some Sobolev space with corner at its crest. The stability and interaction of peakons were discussed in several references $[1,3,8,9,26]$. 
As extension of the $\mathrm{CH}$ peakon equation, other integrable peakon models have also been found, such as the Degasperis-Procesi (DP) equation $[11,12,29]$

$$
m_{t}+3 m u_{x}+m_{x} u=0, \quad m=u-u_{x x}
$$

the cubic nonlinear peakon equation $[15,20,32,34,35]$

$$
m_{t}=\frac{1}{2}\left[m\left(u^{2}-u_{x}^{2}\right)\right]_{x}, \quad m=u-u_{x x}
$$

and the Novikov's cubic nonlinear equation $[25,31]$

$$
m_{t}=u^{2} m_{x}+3 u u_{x} m, \quad m=u-u_{x x}
$$

Then, a naturally interesting theme is to study integrable multi-component peakon equations. For example, in $[6,14,24,32]$ the authors proposed a two-component generalization of the $\mathrm{CH}$ equation. In $[21,38,42,43]$, two-component extensions of the cubic nonlinear equations (1.3) and (1.4) were investigated, while in $[17,22,36]$ three-component extensions of the $\mathrm{CH}$ equation are derived.

In this paper, we propose the following three-component system

$$
\left\{\begin{aligned}
m_{11, t}= & \frac{1}{2}\left[m_{11}\left(u_{11}^{2}-u_{11, x}^{2}+u_{12} u_{21}-u_{12, x} u_{21, x}\right)\right]_{x} \\
& +\frac{1}{2} m_{12}\left(u_{11, x} u_{21}-u_{11} u_{21, x}\right)-\frac{1}{2} m_{21}\left(u_{11} u_{12, x}-u_{11, x} u_{12}\right) \\
m_{12, t}= & \frac{1}{2}\left[m_{12}\left(u_{11}^{2}-u_{11, x}^{2}+u_{12} u_{21}-u_{12, x} u_{21, x}\right)\right]_{x} \\
& +m_{11}\left(u_{11} u_{12, x}-u_{11, x} u_{12}\right)+\frac{1}{2} m_{12}\left(u_{12, x} u_{21}-u_{12} u_{21, x}\right) \\
m_{21, t}= & \frac{1}{2}\left[m_{21}\left(u_{11}^{2}-u_{11, x}^{2}+u_{12} u_{21}-u_{12, x} u_{21, x}\right)\right]_{x} \\
& +m_{11}\left(u_{11} u_{21, x}-u_{11, x} u_{21}\right)+\frac{1}{2} m_{21}\left(u_{12} u_{21, x}-u_{12, x} u_{21}\right) \\
m_{11}= & u_{11}-u_{11, x x}, \quad m_{12}=u_{12}-u_{12, x x}, \quad m_{21}=u_{21}-u_{21, x x}
\end{aligned}\right.
$$

Apparently, this system is reduced to the $\mathrm{CH}$ equation (1.1) as $u_{11}=0, u_{21}=2$ and to the cubic nonlinear $\mathrm{CH}$ equation (1.3) as $u_{12}=u_{21}=0$. Therefore, it is a three-component formation based on the $\mathrm{CH}$ equation (1.1) and the cubic nonlinear $\mathrm{CH}$ equation (1.3), and we may call equation (1.5) the $3 \mathrm{CH}$ model. We show that the $3 \mathrm{CH}$ system is Hamiltonian and possesses a Lax pair and infinitely many conservation laws. Furthermore, this three-component system admits the single peakon of traveling wave type as well as multi-peakon solutions. Additionally, we pay attention to reductions of the $3 \mathrm{CH}$ system so that a new integrable perturbed $\mathrm{CH}$ equation with cubic nonlinearity is generated to possess peakon solutions.

The whole paper is organized as follows. In section 2, a Lax pair, Hamiltonian structure, and conservation laws of equation (1.5) are presented. In section 3, the single-peakon and multi-peakon solutions of equation (1.5) are given. Section 4 studies the reductions of system (1.5). Some conclusions and discussions are described in section 5. 


\section{Lax pair, Hamiltonian form and conservation laws}

Let us first introduce the $s l(2)$ valued matrices $u$ and $m$ as follows:

$$
u=\left(\begin{array}{cc}
u_{11} & u_{12} \\
u_{21} & -u_{11}
\end{array}\right), \quad m=\left(\begin{array}{cc}
u_{11}-u_{11, x x} & u_{12}-u_{12, x x} \\
u_{21}-u_{21, x x} & -u_{11}+u_{11, x x}
\end{array}\right) \triangleq\left(\begin{array}{cc}
m_{11} & m_{12} \\
m_{21} & -m_{11}
\end{array}\right)
$$

Using this notation, equation (1.5) can be expressed in a nice matrix equation form

$$
m_{t}=\frac{1}{2}\left[m\left(u^{2}-u_{x}^{2}\right)\right]_{x}+\frac{1}{4}\left[m\left(u u_{x}-u_{x} u\right)-\left(u u_{x}-u_{x} u\right) m\right], \quad m=u-u_{x x}
$$

where $u$ and $m$ are the $s l(2)$ matrices (2.1).

Consider a pair of linear spectral problems

$$
\phi_{x}=U \phi, \quad \phi_{t}=V \phi,
$$

with

$$
\begin{aligned}
\phi & =\left(\phi_{1}, \phi_{2}, \phi_{3}, \phi_{4}\right)^{T} \\
U & =\frac{1}{2}\left(\begin{array}{cc}
-I_{2} & \lambda m \\
\lambda m & I_{2}
\end{array}\right) \triangleq\left(\begin{array}{cc}
U_{11} & U_{12} \\
U_{21} & U_{22}
\end{array}\right), \\
V & =\frac{1}{2}\left(\begin{array}{cc}
\lambda-2 & I_{2}-\frac{1}{2}\left(u^{2}-u_{x}^{2}+u u_{x}-u_{x} u\right)-\lambda^{-1}\left(u-u_{x}\right)+\frac{1}{2} \lambda m\left(u^{2}-u_{x}^{2}\right) \\
-\lambda^{-1}\left(u+u_{x}\right)+\frac{1}{2} \lambda m\left(u^{2}-u_{x}^{2}\right)-\lambda^{-2} I_{2}+\frac{1}{2}\left(u^{2}-u_{x}^{2}+u_{x} u-u u_{x}\right)
\end{array}\right) \\
& \triangleq\left(\begin{array}{ll}
V_{11} & V_{12} \\
V_{21} & V_{22}
\end{array}\right)
\end{aligned}
$$

where $\lambda$ is a spectral parameter, $I_{2}$ is the $2 \times 2$ identity matrix, and $u$ and $m$ are the $s l(2)$ valued matrices (2.1).

The compatibility condition of (2.3) generates

$$
U_{t}-V_{x}+[U, V]=0
$$

Substituting the expressions of $U$ and $V$ given by (2.4) into (2.5), we find that (2.5) is nothing but the matrix equation (2.2). Hence, (2.3) exactly gives the Lax pair of equation (1.5).

Motivated by the $2 \times 2$ Hamiltonian operators we proposed in [42] and by a tough guesswork, we figure out the following $3 \times 3$ operator

$$
J=\left(\begin{array}{lll}
J_{11} & J_{12} & J_{13} \\
J_{21} & J_{22} & J_{23} \\
J_{31} & J_{32} & J_{33}
\end{array}\right)
$$


where

$$
\begin{aligned}
& J_{11}=\partial m_{11} \partial^{-1} m_{11} \partial+\frac{1}{2} m_{12} \partial^{-1} m_{21}+\frac{1}{2} m_{21} \partial^{-1} m_{12}, \\
& J_{12}=\partial m_{11} \partial^{-1} m_{12} \partial-m_{12} \partial^{-1} m_{11}, \\
& J_{13}=\partial m_{11} \partial^{-1} m_{21} \partial-m_{21} \partial^{-1} m_{11}, \\
& J_{21}=-J_{12}^{*}=\partial m_{12} \partial^{-1} m_{11} \partial-m_{11} \partial^{-1} m_{12}, \\
& J_{22}=\partial m_{12} \partial^{-1} m_{12} \partial-m_{12} \partial^{-1} m_{12}, \\
& J_{23}=\partial m_{12} \partial^{-1} m_{21} \partial+2 m_{11} \partial^{-1} m_{11}+m_{12} \partial^{-1} m_{21}, \\
& J_{31}=-J_{13}^{*}=\partial m_{21} \partial^{-1} m_{11} \partial-m_{11} \partial^{-1} m_{21}, \\
& J_{32}=-J_{23}^{*}=\partial m_{21} \partial^{-1} m_{12} \partial+2 m_{11} \partial^{-1} m_{11}+m_{21} \partial^{-1} m_{12}, \\
& J_{33}=\partial m_{21} \partial^{-1} m_{21} \partial-m_{21} \partial^{-1} m_{21} .
\end{aligned}
$$

It is easy to check that $J$ is skew-symmetric. By a direct but tedious calculation, we can prove the Jacobi identity

$$
\left\langle\alpha, J^{\prime}[J \beta] \gamma\right\rangle+\left\langle\beta, J^{\prime}[J \gamma] \alpha\right\rangle+\left\langle\gamma, J^{\prime}[J \alpha] \beta\right\rangle=0,
$$

where the prime-sign stands for the Gâteaux derivative of an operator [20], and

$$
\alpha=\left(\alpha_{1}, \alpha_{2}, \alpha_{3}\right)^{T}, \quad \beta=\left(\beta_{1}, \beta_{2}, \beta_{3}\right)^{T}, \quad \gamma=\left(\gamma_{1}, \gamma_{2}, \gamma_{3}\right)^{T},
$$

are arbitrary testing three-dimensional vectors. Thus $J$ is a Hamiltonian operator.

Proposition 2.1. Equation (1.5) can be rewritten in the following Hamiltonian form

$$
\left(m_{11, t}, m_{12, t}, m_{21, t}\right)^{T}=J\left(\frac{\delta H}{\delta m_{11}}, \frac{\delta H}{\delta m_{12}}, \frac{\delta H}{\delta m_{21}}\right)^{T},
$$

where $J$ is given by (2.6), and

$$
H_{1}=\frac{1}{2} \int_{-\infty}^{+\infty}\left(u_{11}^{2}+u_{12} u_{21}+u_{11, x}^{2}+u_{12, x} u_{21, x}\right) d x .
$$

We believe that the $3 \mathrm{CH}$ equation (1.5) could be cast into a bi-Hamiltonian system. But we didn't find another Hamiltonian operator yet that is compatible with the Hamiltonian operator (2.6). This is mainly due to complexity of the $3 \mathrm{CH}$ system (1.5) with three-component.

Next, let us construct conservation laws of equation (1.5) with the method developed in $[40,41]$. We consider

$$
\left(\begin{array}{l}
\Phi_{1} \\
\Phi_{2}
\end{array}\right)_{x}=\left(\begin{array}{ll}
U_{11} & U_{12} \\
U_{21} & U_{22}
\end{array}\right)\left(\begin{array}{l}
\Phi_{1} \\
\Phi_{2}
\end{array}\right), \quad\left(\begin{array}{l}
\Phi_{1} \\
\Phi_{2}
\end{array}\right)_{t}=\left(\begin{array}{ll}
V_{11} & V_{12} \\
V_{21} & V_{22}
\end{array}\right)\left(\begin{array}{l}
\Phi_{1} \\
\Phi_{2}
\end{array}\right)
$$

where $\Phi_{1}, \Phi_{2}, U_{i j}$ and $V_{i j}, 1 \leq i, j \leq 2$, are all $2 \times 2$ matrices. Let $\Omega=\Phi_{2} \Phi_{1}^{-1}$, then we may check that $\Omega$ satisfies the following matrix Riccati equation

$$
\Omega_{x}=U_{21}+U_{22} \Omega-\Omega U_{11}-\Omega U_{12} \Omega .
$$

From the compatibility condition of (2.12), we arrive at the conservation law

$$
\left[\operatorname{tr}\left(U_{11}+U_{12} \Omega\right)\right]_{t}=\left[\operatorname{tr}\left(V_{11}+V_{12} \Omega\right)\right]_{x},
$$


where $\operatorname{tr}(A)$ denotes the trace of a matrix $A$.

Substituting the expressions $U_{i j}$ and $V_{i j}, 1 \leq i, j \leq 2$, given by (2.4) into (2.13) and (2.14), we immediately obtain the Riccati equation and conservation law for our equation (1.5)

$$
\begin{aligned}
& \Omega_{x}=\frac{1}{2} \lambda m+\Omega-\frac{1}{2} \lambda \Omega m \Omega, \\
& {[\operatorname{tr}(m \Omega)]_{t}=\left[\operatorname{tr}\left(-\lambda^{-2}\left(u-u_{x}\right) \Omega-\frac{1}{2} \lambda^{-1}\left(u^{2}-u_{x}^{2}\right)+\frac{1}{2} m\left(u^{2}-u_{x}^{2}\right) \Omega\right)\right]_{x} .}
\end{aligned}
$$

Equation (2.16) shows that $\operatorname{tr}(m \Omega)$ is a generating function of the conserved densities. To derive the explicit forms of conserved densities, we expand $m \Omega$ in terms of negative powers of $\lambda$ as below:

$$
m \Omega=\sum_{j=0}^{\infty} \omega_{j} \lambda^{-j}
$$

Substituting (2.17) into (2.15) and equating the coefficients of powers of $\lambda$, we arrive at

$$
\begin{aligned}
\omega_{0} & =\left(m_{11}^{2}+m_{12} m_{21}\right)^{\frac{1}{2}} I_{2}, \quad \omega_{1}=\omega_{0}^{-1}\left[\omega_{0}-m\left(m^{-1} \omega_{0}\right)_{x}\right], \\
\omega_{j+1} & =\omega_{0}^{-1}\left[\omega_{j}-\frac{1}{2} \sum_{i+k=j+1,1 \leq i, k \leq j} \omega_{i} \omega_{k}-m\left(m^{-1} \omega_{j}\right)_{x}\right], \quad j \geq 1 .
\end{aligned}
$$

Inserting (2.17) and (2.18) into (2.16), we finally obtain the following infinitely many conserved densities $\rho_{j}$ and the associated fluxes $F_{j}$ :

$$
\begin{aligned}
\rho_{0} & =\operatorname{tr}\left(\omega_{0}\right)=2\left(m_{11}^{2}+m_{12} m_{21}\right)^{\frac{1}{2}}, \\
F_{0} & =\frac{1}{2} \operatorname{tr}\left[\left(u^{2}-u_{x}^{2}\right) \omega_{0}\right]=\left(u_{11}^{2}-u_{11, x}^{2}+u_{12} u_{21}-u_{12, x} u_{21, x}\right)\left(m_{11}^{2}+m_{12} m_{21}\right)^{\frac{1}{2}}, \\
\rho_{1} & =\operatorname{tr}\left(\omega_{1}\right), \quad F_{1}=\frac{1}{2} \operatorname{tr}\left[-\left(u^{2}-u_{x}^{2}\right)+\left(u^{2}-u_{x}^{2}\right) \omega_{1}\right], \\
\rho_{2} & =\operatorname{tr}\left(\omega_{2}\right), \quad F_{2}=\operatorname{tr}\left[-\left(u-u_{x}\right) m^{-1} \omega_{0}+\frac{1}{2}\left(u^{2}-u_{x}^{2}\right) \omega_{2}\right], \\
\rho_{j+1} & =\operatorname{tr}\left(\omega_{j+1}\right), \quad F_{j+1}=\operatorname{tr}\left[-\left(u-u_{x}\right) m^{-1} \omega_{j-1}+\frac{1}{2}\left(u^{2}-u_{x}^{2}\right) \omega_{j+1}\right], \quad j \geq 2,
\end{aligned}
$$

where $\omega_{j}$ is given by (2.18).

\section{Peakon solutions}

Let us suppose that a single peakon solution of (1.5) has the following form

$$
u_{11}=c_{11} e^{-|x-c t|}, \quad u_{12}=c_{12} e^{-|x-c t|}, \quad u_{21}=c_{21} e^{-|x-c t|},
$$

where the constants $c_{11}, c_{12}$ and $c_{21}$ are to be determined. The first order derivatives of $u_{11}, u_{12}$ and $u_{21}$ do not exist at $x=c t$. Thus (3.1) can not be a solution of equation (1.5) in the classical sense. 
However, with the help of distribution theory we have

$$
\begin{array}{ll}
u_{11, x}=-c_{11} \operatorname{sgn}(x-c t) e^{-|x-c t|}, & m_{11}=2 c_{11} \delta(x-c t), \\
u_{12, x}=-c_{12} \operatorname{sgn}(x-c t) e^{-|x-c t|}, & m_{12}=2 c_{12} \delta(x-c t), \\
u_{21, x}=-c_{21} \operatorname{sgn}(x-c t) e^{-|x-c t|}, & m_{21}=2 c_{21} \delta(x-c t) .
\end{array}
$$

Integrating the equation (1.5) against an arbitrary test function $\phi(x, t)$ with compact support, then moving the derivatives to $\phi(x, t)$, and finally substituting (3.1) and (3.2) into the resulting equations, the left hand side of the first equation in (1.5) produces

$$
\begin{aligned}
& \int_{-\infty}^{+\infty} \int_{-\infty}^{+\infty} m_{11, t} \phi(x, t) d x d t \\
= & -c c_{11} \int_{-\infty}^{+\infty} \int_{-\infty}^{+\infty}\left(E-E_{x x}\right)_{x} \phi(x, t) d x d t \\
= & c c_{11} \int_{-\infty}^{+\infty} \int_{-\infty}^{+\infty}\left(E \phi_{x}(x, t)-E \phi_{x x x}(x, t)\right) d x d t \\
= & c c_{11} \int_{-\infty}^{+\infty}\left[\int_{-\infty}^{c t} e^{x-c t} \phi_{x}(x, t) d x+\int_{c t}^{+\infty} e^{-(x-c t)} \phi_{x}(x, t) d x\right. \\
& \left.-\int_{-\infty}^{c t} e^{x-c t} \phi_{x x x}(x, t) d x-\int_{c t}^{+\infty} e^{-(x-c t)} \phi_{x x x}(x, t) d x\right] d t \\
= & c c_{11} \int_{-\infty}^{+\infty}\left[\int_{-\infty}^{c t} e^{x-c t} \phi_{x}(x, t) d x+\int_{c t}^{+\infty} e^{-(x-c t)} \phi_{x}(x, t) d x\right. \\
& \left.+\int_{-\infty}^{c t} e^{x-c t} \phi_{x x}(x, t) d x-\int_{c t}^{+\infty} e^{-(x-c t)} \phi_{x x}(x, t) d x\right] d t \\
= & c c_{11} \int_{-\infty}^{+\infty}\left[\int_{-\infty}^{c t} e^{x-c t} \phi_{x}(x, t) d x+\int_{c t}^{+\infty} e^{-(x-c t)} \phi_{x}(x, t) d x\right. \\
& \left.+2 \phi^{\prime}(c t, t)-\int_{-\infty}^{c t} e^{x-c t} \phi_{x}(x, t) d x-\int_{c t}^{+\infty} e^{-(x-c t)} \phi_{x}(x, t) d x\right] d t \\
= & 2 c c_{11} \int_{-\infty}^{+\infty} \phi^{\prime}(c t, t) d t,
\end{aligned}
$$

where the notations $E=e^{-|x-c t|}$ and $\phi^{\prime}(c t, t)=\left.\phi_{x}(x, t)\right|_{x=c t}$. We split the right hand side of the first equation in (1.5) into the following four parts

$$
\begin{aligned}
& -\frac{1}{2} \int_{-\infty}^{+\infty} \int_{-\infty}^{+\infty} m_{11}\left(u_{11}^{2}+u_{12} u_{21}\right) \phi_{x}(x, t) d x d t \\
& +\frac{1}{2} \int_{-\infty}^{+\infty} \int_{-\infty}^{+\infty} m_{11}\left(u_{11, x}^{2}+u_{12, x} u_{21, x}\right) \phi_{x}(x, t) d x d t \\
& +\frac{1}{2} \int_{-\infty}^{+\infty} \int_{-\infty}^{+\infty} m_{12}\left(u_{11, x} u_{21}-u_{11} u_{21, x}\right) \phi(x, t) d x d t \\
& -\frac{1}{2} \int_{-\infty}^{+\infty} \int_{-\infty}^{+\infty} m_{21}\left(u_{11} u_{12, x}-u_{11, x} u_{12}\right) \phi(x, t) d x d t \\
& \triangleq I_{1}+I_{2}+I_{3}+I_{4} .
\end{aligned}
$$


We compute $I_{1}$ as follows

$$
\begin{aligned}
I_{1}= & -\frac{1}{2} c_{11}\left(c_{11}^{2}+c_{12} c_{21}\right) \int_{-\infty}^{+\infty} \int_{-\infty}^{+\infty}\left(E-E_{x x}\right) E^{2} \phi_{x}(x, t) d x d t \\
= & -\frac{1}{2} c_{11}\left(c_{11}^{2}+c_{12} c_{21}\right) \int_{-\infty}^{+\infty} \int_{-\infty}^{+\infty}\left[E^{3}-\left(E^{2} E_{x}\right)_{x}+2 E E_{x}^{2}\right] \phi_{x}(x, t) d x d t \\
= & -\frac{1}{2} c_{11}\left(c_{11}^{2}+c_{12} c_{21}\right) \int_{-\infty}^{+\infty} \int_{-\infty}^{+\infty}\left[\left(E^{3}+2 E E_{x}^{2}\right) \phi_{x}(x, t)+E^{2} E_{x} \phi_{x x}(x, t)\right] d x d t \\
= & -\frac{1}{2} c_{11}\left(c_{11}^{2}+c_{12} c_{21}\right) \int_{-\infty}^{+\infty}\left[3 \int_{-\infty}^{c t} e^{3(x-c t)} \phi_{x}(x, t) d x+3 \int_{c t}^{+\infty} e^{-3(x-c t)} \phi_{x}(x, t) d x\right. \\
& \left.+\int_{-\infty}^{c t} e^{3(x-c t)} \phi_{x x}(x, t) d x-\int_{c t}^{+\infty} e^{-3(x-c t)} \phi_{x x}(x, t) d x\right] d t \\
= & -\frac{1}{2} c_{11}\left(c_{11}^{2}+c_{12} c_{21}\right) \int_{-\infty}^{+\infty}\left[3 \int_{-\infty}^{c t} e^{3(x-c t)} \phi_{x}(x, t) d x+3 \int_{c t}^{+\infty} e^{-3(x-c t)} \phi_{x}(x, t) d x\right. \\
& \left.+2 \phi^{\prime}(c t, t)-3 \int_{-\infty}^{c t} e^{3(x-c t)} \phi_{x}(x, t) d x-3 \int_{c t}^{+\infty} e^{-3(x-c t)} \phi_{x}(x, t) d x\right] d t \\
= & -c_{11}\left(c_{11}^{2}+c_{12} c_{21}\right) \int_{-\infty}^{+\infty} \phi^{\prime}(c t, t) d t .
\end{aligned}
$$

In a similar manner, we obtain

$$
\begin{aligned}
& I_{2}=\frac{1}{3} c_{11}\left(c_{11}^{2}+c_{12} c_{21}\right) \int_{-\infty}^{+\infty} \phi^{\prime}(c t, t) d t, \\
& I_{3}=I_{4}=0 .
\end{aligned}
$$

Thus it follows from the first equation in (1.5) that

$$
c_{11}^{2}+c_{12} c_{21}=-3 c .
$$

By similar calculations, we find that the second and the third equations in (1.5) also give rise to (3.3). Hence the single peakon solution becomes

$$
u_{11}=c_{11} e^{-\left|x+\frac{c_{11}^{2}+c_{12} c_{21}}{3} t\right|}, \quad u_{12}=c_{12} e^{-\left|x+\frac{c_{11}^{2}+c_{12} c_{21}}{3} t\right|}, \quad u_{21}=c_{21} e^{-\left|x+\frac{c_{11}^{2}+c_{12} c_{21}}{3} t\right|} .
$$

In general, we assume that an $N$-peakon solution has the following form

$$
u_{11}=\sum_{j=1}^{N} p_{j}(t) e^{-\left|x-q_{j}(t)\right|}, u_{12}=\sum_{j=1}^{N} r_{j}(t) e^{-\left|x-q_{j}(t)\right|}, u_{21}=\sum_{j=1}^{N} s_{j}(t) e^{-\left|x-q_{j}(t)\right|} .
$$

In the distribution sense, we have

$$
\begin{array}{ll}
u_{11, x}=-\sum_{j=1}^{N} p_{j} \operatorname{sgn}\left(x-q_{j}\right) e^{-\left|x-q_{j}\right|}, & m_{11}=2 \sum_{j=1}^{N} p_{j} \delta\left(x-q_{j}\right), \\
u_{12, x}=-\sum_{j=1}^{N} r_{j} \operatorname{sgn}\left(x-q_{j}\right) e^{-\left|x-q_{j}\right|}, & m_{12}=2 \sum_{j=1}^{N} r_{j} \delta\left(x-q_{j}\right), \\
u_{21, x}=-\sum_{j=1}^{N} s_{j} \operatorname{sgn}\left(x-q_{j}\right) e^{-\left|x-q_{j}\right|}, & m_{21}=2 \sum_{j=1}^{N} s_{j} \delta\left(x-q_{j}\right) .
\end{array}
$$


Substituting (3.5) and (3.6) into (1.5) and integrating against test function with compact support, we obtain the $N$-peakon dynamic system as follows:

$$
\left\{\begin{aligned}
p_{j, t}= & \frac{1}{2} r_{j} \sum_{i, k=1}^{N} p_{i} s_{k}\left(\operatorname{sgn}\left(q_{j}-q_{k}\right)-\operatorname{sgn}\left(q_{j}-q_{i}\right)\right) e^{-\left|q_{j}-q_{k}\right|-\left|q_{j}-q_{i}\right|} \\
& -\frac{1}{2} s_{j} \sum_{i, k=1}^{N} p_{i} r_{k}\left(\operatorname{sgn}\left(q_{j}-q_{i}\right)-\operatorname{sgn}\left(q_{j}-q_{k}\right)\right) e^{-\left|q_{j}-q_{k}\right|-\left|q_{j}-q_{i}\right|}, \\
r_{j, t}= & p_{j} \sum_{i, k=1}^{N} p_{i} r_{k}\left(\operatorname{sgn}\left(q_{j}-q_{i}\right)-\operatorname{sgn}\left(q_{j}-q_{k}\right)\right) e^{-\left|q_{j}-q_{k}\right|-\left|q_{j}-q_{i}\right|} \\
& +\frac{1}{2} r_{j} \sum_{i, k=1}^{N} r_{i} s_{k}\left(\operatorname{sgn}\left(q_{j}-q_{k}\right)-\operatorname{sgn}\left(q_{j}-q_{i}\right)\right) e^{-\left|q_{j}-q_{k}\right|-\left|q_{j}-q_{i}\right|}, \\
s_{j, t}= & p_{j} \sum_{i, k=1}^{N} p_{i} s_{k}\left(\operatorname{sgn}\left(q_{j}-q_{i}\right)-\operatorname{sgn}\left(q_{j}-q_{k}\right)\right) e^{-\left|q_{j}-q_{k}\right|-\left|q_{j}-q_{i}\right|} \\
& -\frac{1}{2} s_{j} \sum_{i, k=1}^{N} r_{i} s_{k}\left(\operatorname{sgn}\left(q_{j}-q_{k}\right)-\operatorname{sgn}\left(q_{j}-q_{i}\right)\right) e^{-\left|q_{j}-q_{k}\right|-\left|q_{j}-q_{i}\right|}, \\
q_{j, t}= & \frac{1}{6}\left(p_{j}^{2}+r_{j} s_{j}\right)-\frac{1}{2} \sum_{i, k=1}^{N}\left(p_{i} p_{k}+r_{i} s_{k}\right)\left(1-\operatorname{sgn}\left(q_{j}-q_{i}\right) \operatorname{sgn}\left(q_{j}-q_{k}\right)\right) e^{-\left|q_{j}-q_{i}\right|-\left|q_{j}-q_{k}\right|} .
\end{aligned}\right.
$$

We still do not know whether this system is integrable for $N \geq 2$ with respect to a suitable Poisson structure.

\section{Reductions and a new integrable perturbation equation}

As mentioned above, system (1.5) can be reduced to the $\mathrm{CH}$ equation (1.1) as $u_{11}=0, u_{21}=2$ and to the cubic nonlinear $\mathrm{CH}$ equation (1.3) as $u_{12}=u_{21}=0$. Now we discuss the two-component reductions of system (1.5).

\section{Example 1. The integrable two-component system proposed in [42]}

As $u_{11}=0$, equation (1.5) is reduced to a two-component equation

$$
\left\{\begin{array}{l}
m_{12, t}=\frac{1}{2}\left[m_{12}\left(u_{12} u_{21}-u_{12, x} u_{21, x}\right)\right]_{x}+\frac{1}{2} m_{12}\left(u_{12, x} u_{21}-u_{12} u_{21, x}\right), \\
m_{21, t}=\frac{1}{2}\left[m_{21}\left(u_{12} u_{21}-u_{12, x} u_{21, x}\right)\right]_{x}+\frac{1}{2} m_{21}\left(u_{12} u_{21, x}-u_{12, x} u_{21}\right), \\
m_{12}=u_{12}-u_{12, x x} \\
m_{21}=u_{21}-u_{21, x x}
\end{array}\right.
$$

which is exactly the system we derived in [42]. For the bi-Hamiltonian structure and peakon solutions of this system, one may see [42]. 


\section{Example 2. The integrable two-component system presented in [37]}

As $u_{12}=u_{21}$, equation (1.5) is reduced to a two-component equation

$$
\left\{\begin{array}{l}
m_{11, t}=\frac{1}{2}\left[m_{11}\left(u_{11}^{2}+u_{12}^{2}-u_{11, x}^{2}-u_{12, x}^{2}\right)\right]_{x}+m_{12}\left(u_{11, x} u_{12}-u_{11} u_{12, x}\right), \\
m_{12, t}=\frac{1}{2}\left[m_{12}\left(u_{11}^{2}+u_{12}^{2}-u_{11, x}^{2}-u_{12, x}^{2}\right)\right]_{x}+m_{11}\left(u_{11} u_{12, x}-u_{11, x} u_{12}\right), \\
m_{11}=u_{11}-u_{11, x x}, \\
m_{12}=u_{12}-u_{12, x x},
\end{array}\right.
$$

which was proposed by Qu, Song and Yao in [37]. Here in our paper, we want to derive the peakon solutions to this system. Suppose that an $N$-peakon solution of (4.2) has the form

$$
u_{11}=\sum_{j=1}^{N} p_{j}(t) e^{-\left|x-q_{j}(t)\right|}, u_{12}=\sum_{j=1}^{N} r_{j}(t) e^{-\left|x-q_{j}(t)\right|} .
$$

From (3.7) and the reduction condition $u_{12}=u_{21}$, we immediately arrive at the $N$-peakon dynamic system of (4.2):

$$
\left\{\begin{array}{l}
p_{j, t}=r_{j} \sum_{i, k=1}^{N} p_{i} r_{k}\left(\operatorname{sgn}\left(q_{j}-q_{k}\right)-\operatorname{sgn}\left(q_{j}-q_{i}\right)\right) e^{-\left|q_{j}-q_{k}\right|-\left|q_{j}-q_{i}\right|}, \\
r_{j, t}=p_{j} \sum_{i, k=1}^{N} p_{i} r_{k}\left(\operatorname{sgn}\left(q_{j}-q_{i}\right)-\operatorname{sgn}\left(q_{j}-q_{k}\right)\right) e^{-\left|q_{j}-q_{k}\right|-\left|q_{j}-q_{i}\right|}, \\
q_{j, t}=\frac{1}{6}\left(p_{j}^{2}+r_{j}^{2}\right)-\frac{1}{2} \sum_{i, k=1}^{N}\left(p_{i} p_{k}+r_{i} r_{k}\right)\left(1-\operatorname{sgn}\left(q_{j}-q_{i}\right) \operatorname{sgn}\left(q_{j}-q_{k}\right)\right) e^{-\left|q_{j}-q_{i}\right|-\left|q_{j}-q_{k}\right|} .
\end{array}\right.
$$

For $N=1$, we find that the single-peakon solution reads

$$
u_{11}=c_{1} e^{-\left|x+\frac{c_{1}^{2}+c_{2}^{2}}{3} t\right|}, \quad u_{12}=c_{2} e^{-\left|x+\frac{c_{1}^{2}+c_{2}^{2}}{3} t\right|},
$$

where $c_{1}$ and $c_{2}$ are integration constants. For $N=2$, we may solve (4.4) as

$$
\left\{\begin{array}{l}
q_{1}(t)=-\frac{1}{3} A_{1}^{2} t+\frac{3 A_{1} A_{2} \cos \left(A_{3}-A_{4}\right)}{\left|A_{1}^{2}-A_{2}^{2}\right|} \operatorname{sgn}(t)\left(e^{-\frac{1}{3}\left|\left(A_{1}^{2}-A_{2}^{2}\right) t\right|}-1\right), \\
q_{2}(t)=-\frac{1}{3} A_{2}^{2} t+\frac{3 A_{1} A_{2} \cos \left(A_{3}-A_{4}\right)}{\left|A_{1}^{2}-A_{2}^{2}\right|} \operatorname{sgn}(t)\left(e^{-\frac{1}{3}\left|\left(A_{1}^{2}-A_{2}^{2}\right) t\right|}-1\right), \\
p_{1}(t)=A_{1} \sin \left(\frac{3 A_{1} A_{2} \sin \left(A_{3}-A_{4}\right)}{A_{1}^{2}-A_{2}^{2}} e^{-\frac{1}{3}\left|\left(A_{1}^{2}-A_{2}^{2}\right) t\right|}+A_{3}\right), \\
p_{2}(t)=A_{2} \sin \left(\frac{3 A_{1} A_{2} \sin \left(A_{3}-A_{4}\right)}{A_{1}^{2}-A_{2}^{2}} e^{-\frac{1}{3}\left|\left(A_{1}^{2}-A_{2}^{2}\right) t\right|}+A_{4}\right), \\
r_{1}(t)=A_{1} \cos \left(\frac{3 A_{1} A_{2} \sin \left(A_{3}-A_{4}\right)}{A_{1}^{2}-A_{2}^{2}} e^{-\frac{1}{3}\left|\left(A_{1}^{2}-A_{2}^{2}\right) t\right|}+A_{3}\right), \\
r_{2}(t)=A_{2} \cos \left(\frac{3 A_{1} A_{2} \sin \left(A_{3}-A_{4}\right)}{A_{1}^{2}-A_{2}^{2}} e^{-\frac{1}{3}\left|\left(A_{1}^{2}-A_{2}^{2}\right) t\right|}+A_{4}\right),
\end{array}\right.
$$

where $A_{1}, \cdots, A_{4}$ are integration constants. In particular, letting $A_{1}=1, A_{2}=2, A_{3}=0$ and $A_{4}=\frac{\pi}{6}$, we obtain the two-peakon solution of (4.2)

$$
\left\{\begin{array}{l}
u_{11}=\sin \left(e^{-|t|}\right) e^{-\left|x-q_{1}(t)\right|}+2 \sin \left(e^{-|t|}+\frac{\pi}{6}\right) e^{-\left|x-q_{2}(t)\right|} \\
u_{12}=\cos \left(e^{-|t|}\right) e^{-\left|x-q_{1}(t)\right|}+2 \cos \left(e^{-|t|}+\frac{\pi}{6}\right) e^{-\left|x-q_{2}(t)\right|}
\end{array}\right.
$$

with

$$
q_{1}(t)=-\frac{1}{3} t+\sqrt{3} \operatorname{sgn}(t)\left(e^{-|t|}-1\right), \quad q_{2}(t)=-\frac{4}{3} t+\sqrt{3} \operatorname{sgn}(t)\left(e^{-|t|}-1\right) .
$$


The two-peakon collides at $t=0$, since $q_{1}(0)=q_{2}(0)=0$. See Figures 1 and 2 for the two-peakon dynamics of the potentials $u_{11}(x, t)$ and $u_{12}(x, t)$.

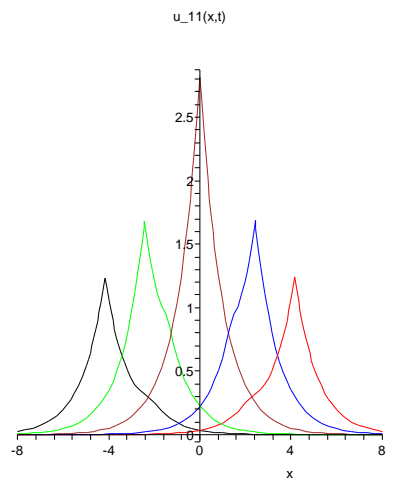

Fig. 1. The two-peakon dynamic for the potential $u_{11}(x, t)$ in (4.7). Red line: $t=-2$; Blue line: $t=-1$; Brown line: $t=0$ (collision); Green line: $t=1$; Black line: $t=2$.

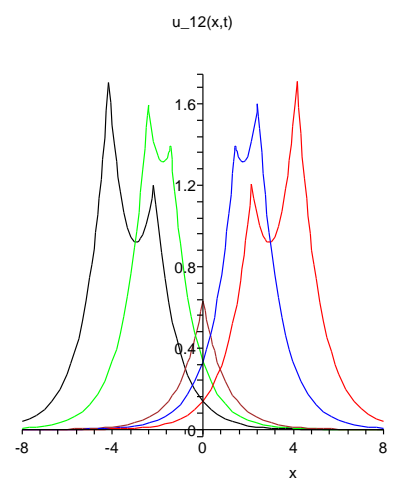

Fig. 2. The two-peakon dynamic for the potential $u_{12}(x, t)$ in (4.7). Red line: $t=-2$; Blue line: $t=-1$; Brown line: $t=0$ (collision); Green line: $t=1$; Black line: $t=2$.

\section{Example 3. A new integrable perturbation of cubic nonlinear CH equation}

As $u_{12}=0$, equation (1.5) is cast into

$$
\left\{\begin{array}{l}
m_{11, t}=\frac{1}{2}\left[m_{11}\left(u_{11}^{2}-u_{11, x}^{2}\right)\right]_{x} \\
m_{21, t}=\frac{1}{2}\left[m_{21}\left(u_{11}^{2}-u_{11, x}^{2}\right)\right]_{x}+m_{11}\left(u_{11} u_{21, x}-u_{11, x} u_{21}\right) \\
m_{11}=u_{11}-u_{11, x x} \\
m_{21}=u_{21}-u_{21, x x}
\end{array}\right.
$$

This equation is different from the standard perturbation equation of the cubic nonlinear $\mathrm{CH}$ system

$$
\left\{\begin{array}{l}
m_{11, t}=\frac{1}{2}\left[m_{11}\left(u_{11}^{2}-u_{11, x}^{2}\right)\right]_{x}, \\
m_{21, t}=\frac{1}{2}\left[m_{21}\left(u_{11}^{2}-u_{11, x}^{2}\right)\right]_{x}+\left[m_{11}\left(u_{11} u_{21}-u_{11, x} u_{21, x}\right)\right]_{x}, \\
m_{11}=u_{11}-u_{11, x x} \\
m_{21}=u_{21}-u_{21, x x}
\end{array}\right.
$$


which can be constructed by a small disturbance (in the sense of $[19,30]$ ) of the cubic nonlinear $\mathrm{CH}$ equation (for detail, see Remark 1 at the end of this example). Thus (4.9) is a new integrable perturbation of the cubic nonlinear $\mathrm{CH}$ equation. It is noticed that by direct calculations one may see that the second potential $u_{21}$ of the standard perturbation equation (4.10) does not admit peakon solution in the form of $u_{21}=p(t) e^{-|x-q(t)|}$. However, we find that our new perturbation equation (4.9) admits peakon solutions. In fact, suppose that an $N$-peakon solution of (4.9) has the form

$$
u_{11}=\sum_{j=1}^{N} p_{j}(t) e^{-\left|x-q_{j}(t)\right|}, u_{21}=\sum_{j=1}^{N} s_{j}(t) e^{-\left|x-q_{j}(t)\right|},
$$

we obtain the $N$-peakon dynamic system of (4.9) as follows:

$$
\left\{\begin{array}{l}
p_{j, t}=0 \\
q_{j, t}=\frac{1}{6} p_{j}^{2}-\frac{1}{2} \sum_{i, k=1}^{N} p_{i} p_{k}\left(1-\operatorname{sgn}\left(q_{j}-q_{i}\right) \operatorname{sgn}\left(q_{j}-q_{k}\right)\right) e^{-\left|q_{j}-q_{i}\right|-\left|q_{j}-q_{k}\right|} \\
s_{j, t}=p_{j} \sum_{i, k=1}^{N} p_{i} s_{k}\left(\operatorname{sgn}\left(q_{j}-q_{i}\right)-\operatorname{sgn}\left(q_{j}-q_{k}\right)\right) e^{-\left|q_{j}-q_{k}\right|-\left|q_{j}-q_{i}\right|} .
\end{array}\right.
$$

For $N=1$, we find that the single-peakon solution takes the form

$$
u_{11}=\sqrt{-3 c} e^{-|x-c t|}, \quad u_{21}=c_{21} e^{-|x-c t|},
$$

where $c_{21}$ is an arbitrary constant.

For $N=2$, (4.12) becomes

$$
\left\{\begin{array}{l}
p_{1, t}=p_{2, t}=0 \\
q_{1, t}=-\frac{1}{3} p_{1}^{2}-p_{1} p_{2} e^{-\left|q_{1}-q_{2}\right|} \\
q_{2, t}=-\frac{1}{3} p_{2}^{2}-p_{1} p_{2} e^{-\left|q_{1}-q_{2}\right|} \\
s_{1, t}=p_{1}\left(p_{2} s_{1}-p_{1} s_{2}\right) \operatorname{sgn}\left(q_{1}-q_{2}\right) e^{-\left|q_{1}-q_{2}\right|} \\
s_{2, t}=p_{2}\left(p_{2} s_{1}-p_{1} s_{2}\right) \operatorname{sgn}\left(q_{1}-q_{2}\right) e^{-\left|q_{1}-q_{2}\right|}
\end{array}\right.
$$

From the first equation of (4.14), we obtain

$$
p_{1}=A_{1}, \quad p_{2}=A_{2},
$$

where $A_{1}$ and $A_{2}$ are integration constants. Let us set $0<A_{1}<A_{2}$. Then from (4.14), we arrive at

$$
\left\{\begin{array}{l}
q_{1}(t)=-\frac{1}{3} A_{1}^{2} t+\frac{3 A_{1} A_{2}}{A_{2}^{2}-A_{1}^{2}} \operatorname{sgn}(t)\left(e^{-\frac{1}{3}\left(A_{2}^{2}-A_{1}^{2}\right)|t|}-1\right) \\
q_{2}(t)=-\frac{1}{3} A_{2}^{2} t+\frac{3 A_{1} A_{2}}{A_{2}^{2}-A_{1}^{2}} \operatorname{sgn}(t)\left(e^{-\frac{1}{3}\left(A_{2}^{2}-A_{1}^{2}\right)|t|}-1\right) \\
s_{1}(t)=\frac{3 A_{1} A_{3}}{A_{1}^{2}-A_{2}^{2}} e^{-\frac{1}{3}\left(A_{2}^{2}-A_{1}^{2}\right)|t|}+A_{4} \\
s_{2}(t)=\frac{1}{A_{1}}\left(A_{2} s_{1}-A_{3}\right)
\end{array}\right.
$$

where $A_{3}$ and $A_{4}$ are integration constants. In particular, taking $A_{1}=-A_{3}=1, A_{2}=2$ and $A_{4}=0$, we obtain the two-peakon solution of (4.9)

$$
u_{11}=e^{-\left|x-q_{1}(t)\right|}+2 e^{-\left|x-q_{2}(t)\right|}, u_{21}=e^{-|t|} e^{-\left|x-q_{1}(t)\right|}+\left(2 e^{-|t|}+1\right) e^{-\left|x-q_{2}(t)\right|},
$$


with

$$
q_{1}(t)=-\frac{1}{3} t+2 \operatorname{sgn}(t)\left(e^{-|t|}-1\right), \quad q_{2}(t)=-\frac{4}{3} t+2 \operatorname{sgn}(t)\left(e^{-|t|}-1\right) .
$$

This two-peakon collides at the moment of $t=0$, since $q_{1}(0)=q_{2}(0)=0$. See Figures 3 and 4 for the two-peakon dynamics of the potentials $u_{11}(x, t)$ and $u_{21}(x, t)$.

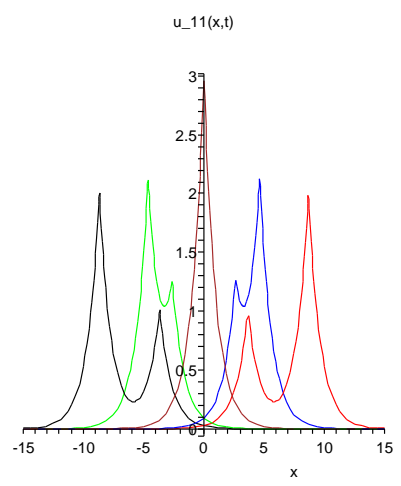

Fig. 3. The two-peakon dynamic for the potential $u_{11}(x, t)$ in (4.17). Red line: $t=-5$; Blue line: $t=-2$; Brown line: $t=0$ (collision); Green line: $t=2$; Black line: $t=5$.

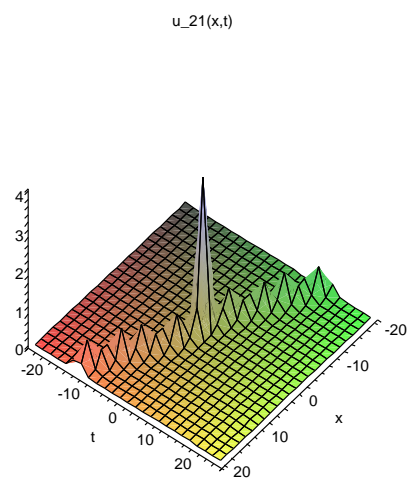

Fig. 4. 3-dimensional graph for the two-peakon dynamic of the potential $u_{21}(x, t)$ in (4.17).

Remark 1. There have been a lot of studies investigating the perturbation equations constructed by a small disturbance of the soliton equations, such as the KdV equation, the MKdV equation, and the AKNS equation (see for example Refs. $[19,30,39]$ ). However, discussions about the perturbation equations of the $\mathrm{CH}$ type equations are rare [27]. This is mainly due to the $\mathrm{CH}$ type equations being of non-evolutionary type. To the best of our knowledge, perturbation equations of the cubic nonlinear $\mathrm{CH}$ system have not been proposed in the literature yet. So, we provide a brief derivation in our paper. Following the paper [30], we make a perturbation expansion

$$
u=\sum_{j=0}^{N} \eta_{j} \varepsilon^{j}, \quad N \geq 1
$$


Replacing $u$ with (4.19) in the cubic nonlinear $\mathrm{CH}$ equation (1.3), and equating the coefficients of powers of $\varepsilon$ (up to $\varepsilon^{N}$ ), we obtain the $n$th perturbation system of the $\mathrm{CH}$ equation with variables $\eta_{0}$, $\eta_{1}, \cdots, \eta_{N}$ :

$$
\left\{\begin{aligned}
m_{0, t} & =\frac{1}{2}\left[m_{0}\left(\eta_{0}^{2}-\eta_{0, x}^{2}\right)\right]_{x}, \\
m_{1, t} & =\left[m_{0}\left(\eta_{0} \eta_{1}-\eta_{0, x} \eta_{1, x}\right)\right]_{x}+\frac{1}{2}\left[m_{1}\left(\eta_{0}^{2}-\eta_{0, x}^{2}\right)\right]_{x}, \\
& \vdots \\
m_{j, t} & =\frac{1}{2}\left[\sum_{k+l=j, k, l \geq 0} m_{k} \sum_{i+h=l, i, h \geq 0}\left(\eta_{i} \eta_{h}-\eta_{i, x} \eta_{h, x}\right)\right]_{x}, \\
& \vdots \\
m_{N, t} & =\frac{1}{2}\left[\sum_{k+l=N, k, l \geq 0} m_{k} \sum_{i+h=l, i, h \geq 0}\left(\eta_{i} \eta_{h}-\eta_{i, x} \eta_{h, x}\right)\right]_{x} \\
m_{0} & =\eta_{0}-\eta_{0, x x}, m_{1}=\eta_{1}-\eta_{1, x x}, \cdots, m_{N}=\eta_{N}-\eta_{N, x x} .
\end{aligned}\right.
$$

For example, as $N=1$, we arrive at the first-order perturbation of cubic nonlinear $\mathrm{CH}$ equation

$$
\left\{\begin{array}{l}
m_{0, t}=\frac{1}{2}\left[m_{0}\left(\eta_{0}^{2}-\eta_{0, x}^{2}\right)\right]_{x}, \\
m_{1, t}=\left[m_{0}\left(\eta_{0} \eta_{1}-\eta_{0, x} \eta_{1, x}\right)\right]_{x}+\frac{1}{2}\left[m_{1}\left(\eta_{0}^{2}-\eta_{0, x}^{2}\right)\right]_{x} \\
m_{0}=\eta_{0}-\eta_{0, x x} \\
m_{1}=\eta_{1}-\eta_{1, x x}
\end{array}\right.
$$

which is nothing but equation (4.10). With suitable adaptations of the techniques used in [30], we may derive the Lax representation, bi-Hamiltonian structure and recursion operator for the resulting perturbation system (4.20). We neglect the details of these results here, since this topic is out of the scope of the present paper. But we would like to stress that, as mentioned before, this perturbation equation does not admit a peakon solution.

\section{Conclusions and discussions}

We have presented an integrable $3 \mathrm{CH}$ peakon system with cubic nonlinearity. The Lax representation, Hamiltonian structure and infinitely many conservation laws of this system are investigated. We also discuss the reductions of this system. In particular, by a reduction we found a new integrable perturbation equation of the cubic nonlinear $\mathrm{CH}$ system. In contrast with the standard perturbation of the cubic nonlinear $\mathrm{CH}$ equation, this new integrable perturbation of the cubic nonlinear $\mathrm{CH}$ equation admits peakon solutions.

\section{ACKNOWLEDGMENTS}

The author Xia was supported by the National Natural Science Foundation of China (Grant No. 11301229), the Natural Science Foundation of the Jiangsu Province (Grant No. BK20130224) and the Natural Science Foundation of the Jiangsu Higher Education Institutions of China (Grant No. 13KJB110009). The author Zhou was supported by the National Natural Science Foundation of 
China (Grant No. 11271168). The author Qiao was partially supported by the National Natural Science Foundation of China (Grant No. 11171295 and 61328103) and also thanks the U.S. Department of Education GAANN project (P200A120256) to support UTPA mathematics graduate program.

\section{References}

[1] M.S. Alber, R. Camassa, Y.N. Fedorov, D.D. Holm and J.E. Marsden, The complex geometry of weak piecewise smooth solutions of integrable nonlinear PDEs of shallow water and Dym type, Commun. Math. Phys. 221 (2001) 197-227.

[2] R. Beals, D. Sattinger and J. Szmigielski, Acoustic scattering and the extended Korteweg-de Vries hierarchy, Adv. Math. 140 (1998) 190-206.

[3] R. Beals, D. Sattinger and J. Szmigielski, Multipeakons and the classical moment problem, Adv. Math. 154 (2000) 229-257.

[4] R. Camassa and D.D. Holm, An integrable shallow water equation with peaked solitons, Phys. Rev. Lett. 71 (1993) 1661-1664.

[5] R. Camassa, D.D. Holm and J.M. Hyman, A new integrable shallow water equation, Adv. Appl. Mech. 31 (1994) 1-33.

[6] M. Chen, S.Q. Liu and Y. Zhang, A two-component generalization of the Camassa-Holm equation and its solutions, Lett. Math. Phys. 75 (2006) 1-15.

[7] A. Constantin, On the inverse spectral problem for the Camassa-Holm equation, J. Funct. Anal. 155 (1998) 352-363.

[8] A. Constantin and W.A. Strauss, Stability of peakons, Comm. Pure Appl. Math. 53 (2000) 603-610.

[9] A. Constantin and W.A. Strauss, Stability of the Camassa-Holm solitons, J. Nonlinear Sci. 12 (2002) 415-422.

[10] A. Constantin, V.S. Gerdjikov and R.I. Ivanov, Inverse scattering transform for the Camassa-Holm equation, Inverse Problems 22 (2006) 2197-2207.

[11] A. Degasperis and M. Procesi, Asymptotic Integrability Symmetry and Perturbation Theory eds A. Degasperis and G. Gaeta (World Scientific, Singapore, 1999) pp. 23-37.

[12] A. Degasperis, D.D. Holm and A.N.W. Hone, A new integrable equation with peakon solitons, Theoret. Math. Phys. 133 (2002) 1463-1474.

[13] H.R. Dullin, G.A. Gottwald and D.D. Holm, An integrable shallow water equation with linear and nonlinear dispersion, Phys. Rev. Lett. 87 (2001) 194501.

[14] G. Falqui, On a Camassa-Holm type equation with two dependent variables, J. Phys. A 39 (2006) 327342.

[15] A.S. Fokas, On a class of physically important integrable equations, Physica D 87 (1995) 145-150.

[16] A.S. Fokas and Q.M. Liu, Asymptotic integrability of water waves, Phys. Rev. Lett. 77 (1996) 23472351.

[17] L. Fontanelli, P. Lorenzoni and M. Pedroni, A three-component extension of the Camassa-Holm hierarchy, Lett. Math. Phys. 78 (2006) 125-137.

[18] B. Fuchssteiner and A.S. Fokas, Symplectic structures, their Bäcklund transformation and hereditary symmetries, Physica D 4 (1981) 47-66.

[19] B. Fuchssteiner, Coupling of completely integrable systems: the perturbation bundle, Applications of analytic and geometric methods to nonlinear differential equations, ed. P.A. Clarkson, (Kluwer, Dordrecht, 1993) pp. 125-138.

[20] B. Fuchssteiner, Some tricks from the symmetry-toolbox for nonlinear equations: generalizations of the Camassa-Holm equation, Physica D 95 (1996) 229-243.

[21] X.G. Geng and B. Xue, An extension of integrable peakon equations with cubic nonlinearity, Nonlinearity 22 (2009) 1847-1856.

[22] X.G. Geng and B. Xue, A three-component generalization of Camassa-Holm equation with N-peakon solutions, Adv. Math. 226 (2011) 827-839.

[23] F. Gesztesy and H. Holden, Algebro-geometric solutions of the Camassa-Holm hierarchy, Rev. Mat. Iberoamericana 19 (2003) 73-142. 
[24] D.D. Holm and R.I. Ivanov, Multi-component generalizations of the $\mathrm{CH}$ equation: geometrical aspects, peakons and numerical examples, J. Phys A: Math. Theor. 43 (2010) 492001.

[25] A.N.W. Hone and J.P. Wang, Integrable peakon equations with cubic nonlinearity, J. Phys. A: Math. Theor. 41 (2008) 372002.

[26] R.S. Johnson, On solutions of the Camassa-Holm equation, Proc. R. Soc. Lond. A 459 (2003) 16871708.

[27] R.A. Kraenkel, M. Senthilvelan and A.I. Zenchuk, On the integrable perturbations of the CamassaHolm equation, J. Math. Phys. 41 (2000) 3160-3169.

[28] P. Lorenzoni and M. Pedroni, On the bi-Hamiltonian structures of the Camassa-Holm and Harry-Dym equations, Int. Math. Res. Not. 75 (2004) 4019-4029.

[29] H. Lundmark and J. Szmigielski, Multi-peakon solutions of the Degasperis-Procesi equation, Inverse Problems 19 (2003) 1241-1245.

[30] W.X. Ma and B. Fuchssteiner, Integrable theory of the perturbation equation, Chaos, Soliton and Fractals 7 (1996) 1227-1250.

[31] V. Novikov, Generalizations of the Camassa-Holm equation, J. Phys. A: Math. Theor. 42 (2009) 342002.

[32] P.J. Olver and P. Rosenau, Tri-Hamiltonian duality between solitons and solitary-wave solutions having compact support, Phys. Rev. E 53 (1996) 1900-1906.

[33] Z.J. Qiao, The Camassa-Holm hierarchy, N-dimensional integrable systems, and algebro-geometric solution on a symplectic submanifold, Commun. Math. Phys. 239 (2003) 309-341.

[34] Z.J. Qiao, A new integrable equation with cuspons and W/M-shape-peaks solitons, J. Math. Phys. 47 (2006) 112701.

[35] Z.J. Qiao, B.Q. Xia and J.B. Li, Integrable system with peakon, weak kink, and kink-peakon interactional solutions, arXiv:1205.2028v2.

[36] C.Z. Qu and Y. Fu, On a new three-component Camassa-Holm equation with peakons, Commun. Theor. Phys. 53 (2010) 223-230.

[37] C.Z. Qu, J.F. Song and R.X. Yao, Multi-component integrable systems and invariant curve flows in certain geometries, SIGMA 9 (2013) 001.

[38] J.F. Song, C.Z. Qu and Z.J. Qiao, A new integrable two-component system with cubic nonlinearity, J. Math. Phys. 52 (2011) 013503.

[39] K.M. Tamizhmani and M. Lakshmanan, Complete integrability of the Korteweg-de Vries equation under perturbation around its solution: Lie-Bäcklund symmetry approach, J. Phys. A: Math. Gen. 16 (1983) 3773-3782.

[40] T. Tsuchida and M. Wadati, The coupled modified Korteweg-de Vries equations, J. Phys. Soc. Jpn. 67 (1998) 1175-1187.

[41] M. Wadati, H. Sanuki and K. Konno, Relationships among inverse method, Bäcklund transformation and an infinite number of conservation laws, Prog. Theor. Phys. 53 (1975) 419-436.

[42] B.Q. Xia and Z.J. Qiao, A new two-component integrable system with peakon and weak kink solutions, arXiv: $1211.5727 \mathrm{v} 2$.

[43] B.Q. Xia, Z.J. Qiao and R.G. Zhou, A synthetical two-component model with peakon solutions, arXiv:1301.3216v2. 